\title{
Erratum
}

\section{Fertility waves, aggregate savings and the rate of interest}

\section{N.S. Blomquist and H. Wijkander}

Department of Economics, Uppsala University, P.O. Box 513, S-75120 Uppsala, Sweden

Some of the tables in the article contain misprints from the original manuscript. We print the correct tables and apologize to our readers for the inconvience.

Table 2. Base simulation. Economic performance when there is a demographic shock in period 2

\begin{tabular}{rlllllllllllr}
\hline$t$ & $s_{t}$ & $s_{t} / w_{t}$ & $K_{t}$ & \multicolumn{1}{c}{$I_{t}$} & $Q_{t}$ & \multicolumn{1}{c}{$I_{t} / Q_{t}$} & $r_{t}$ & $w_{t}$ & $C_{1}^{t}$ & $C_{2}^{t}$ & Comp. \\
\hline 0 & 1.506 & 0.453 & 1.506 & 0.000 & 4.432 & 0.000 & 0.735 & 3.324 & 1.817 & 2.614 & 0.000 \\
1 & 1.479 & 0.445 & 1.506 & -0.027 & 4.432 & -0.006 & 0.855 & 3.324 & 1.844 & 2.744 & -0.270 \\
2 & 1.405 & 0.445 & 1.479 & 0.207 & 5.058 & 0.041 & 0.676 & 3.161 & 1.756 & 2.355 & 0.215 \\
3 & 1.543 & 0.451 & 1.687 & -0.143 & 4.558 & -0.031 & 0.722 & 3.419 & 1.876 & 2.658 & -0.084 \\
4 & 1.514 & 0.453 & 1.543 & -0.029 & 4.458 & -0.006 & 0.733 & 3.344 & 1.829 & 2.624 & -0.018 \\
5 & 1.508 & 0.453 & 1.514 & -0.006 & 4.437 & -0.001 & 0.735 & 3.328 & 1.820 & 2.616 & -0.004 \\
6 & 1.507 & 0.453 & 1.508 & -0.001 & 4.433 & 0.000 & 0.735 & 3.325 & 1.818 & 2.615 & -0.001 \\
7 & 1.507 & 0.453 & 1.507 & 0.000 & 4.432 & 0.000 & 0.735 & 3.324 & 1.817 & 2.614 & 0.000 \\
8 & 1.507 & 0.453 & 1.507 & 0.000 & 4.432 & 0.000 & 0.735 & 3.324 & 1.817 & 2.614 & 0.000 \\
9 & 1.506 & 0.453 & 1.507 & 0.000 & 4.432 & 0.000 & 0.735 & 3.324 & 1.817 & 2.614 & 0.000 \\
10 & 1.506 & 0.453 & 1.506 & 0.000 & 4.432 & 0.000 & 0.735 & 3.324 & 1.817 & 2.614 & 0.000 \\
\hline
\end{tabular}

Assumptions: $\beta=4.0, \alpha=0.25, \varrho=0.5, L_{2}=1.2$ 
Table 3. Alternative simulation. Economic performance when there is a demographic shock in period 2

\begin{tabular}{rlllllllllll}
\hline \multicolumn{1}{l}{$l_{t}$} & $s_{t} / w_{t}$ & $K_{t}$ & \multicolumn{1}{l}{$I_{t}$} & $Q_{t}$ & \multicolumn{1}{c}{$I_{t} / Q_{t}$} & \multicolumn{1}{l}{$r_{t}$} & $w_{t}$ & $C_{1}^{t}$ & $C_{2}^{t}$ & Comp. \\
\hline 0 & 0.897 & 0.307 & 0.897 & 0.000 & 3.893 & 0.000 & 1.085 & 2.919 & 2.023 & 1.870 & 0.000 \\
1 & 0.824 & 0.282 & 0.897 & -0.072 & 3.893 & -0.019 & 1.325 & 2.919 & 2.095 & 1.917 & 0.452 \\
2 & 0.848 & 0.311 & 0.824 & 0.194 & 4.370 & 0.044 & 0.987 & 2.731 & 1.883 & 1.686 & 0.232 \\
3 & 0.929 & 0.308 & 1.018 & -0.089 & 4.018 & -0.022 & 1.057 & 3.013 & 2.085 & 1.910 & -0.082 \\
4 & 0.905 & 0.307 & 0.929 & -0.023 & 3.927 & -0.006 & 1.077 & 2.945 & 2.040 & 1.881 & -0.022 \\
5 & 0.899 & 0.307 & 0.905 & -0.006 & 3.902 & -0.002 & 1.083 & 2.926 & 2.027 & 1.873 & -0.006 \\
6 & 0.898 & 0.307 & 0.899 & -0.002 & 3.895 & 0.000 & 1.084 & 2.921 & 2.024 & 1.871 & -0.002 \\
7 & 0.897 & 0.307 & 0.898 & 0.000 & 3.893 & 0.000 & 1.085 & 2.920 & 2.023 & 1.870 & 0.000 \\
8 & 0.897 & 0.307 & 0.897 & 0.000 & 3.893 & 0.000 & 1.085 & 2.920 & 2.023 & 1.870 & 0.000 \\
9 & 0.897 & 0.307 & 0.897 & 0.000 & 3.893 & 0.000 & 1.085 & 2.919 & 2.023 & 1.870 & 0.000 \\
10 & 0.897 & 0.307 & 0.897 & 0.000 & 3.893 & 0.000 & 1.085 & 2.919 & 2.023 & 1.870 & 0.000 \\
\end{tabular}

Parameter assumptions: $\beta=4.0, \alpha=0.25, \varrho=-0.5, L_{2}=1.2$

Table 4. Economic performance when there are two demographic shocks

\begin{tabular}{rllllllllllr}
\hline$t$ & $s_{t}$ & $s_{t} / w_{t}$ & $K_{t}$ & \multicolumn{1}{c}{$I_{t}$} & $Q_{t}$ & \multicolumn{1}{c}{$I_{t} / Q_{t}$} & $r_{t}$ & $w_{t}$ & $C_{1}^{t}$ & $C_{2}^{t}$ & Comp. \\
\hline 0 & 1.506 & 0.453 & 1.506 & 0.000 & 4.432 & 0.000 & 0.735 & 3.324 & 1.817 & 2.614 & 0.000 \\
1 & 1.479 & 0.445 & 1.506 & -0.027 & 4.432 & -0.006 & 0.855 & 3.324 & 1.844 & 2.744 & -0.270 \\
2 & 1.405 & 0.445 & 1.479 & 0.207 & 5.058 & 0.041 & 0.676 & 3.161 & 1.756 & 2.355 & 0.215 \\
3 & 1.515 & 0.443 & 1.687 & -0.172 & 4.558 & -0.038 & 0.840 & 3.419 & 1.904 & 2.787 & -0.354 \\
4 & 1.413 & 0.444 & 1.515 & 0.180 & 5.088 & 0.035 & 0.673 & 3.180 & 1.767 & 2.363 & 0.199 \\
5 & 1.545 & 0.451 & 1.695 & -0.150 & 4.564 & -0.033 & 0.722 & 3.423 & 1.878 & 2.660 & -0.088 \\
6 & 1.515 & 0.453 & 1.545 & -0.030 & 4.459 & -0.007 & 0.732 & 3.345 & 1.830 & 2.624 & -0.018 \\
7 & 1.508 & 0.453 & 1.515 & -0.006 & 4.437 & -0.001 & 0.735 & 3.328 & 1.820 & 2.616 & -0.004 \\
8 & 1.507 & 0.453 & 1.508 & -0.001 & 4.433 & 0.000 & 0.735 & 3.325 & 1.818 & 2.615 & -0.001 \\
9 & 1.507 & 0.453 & 1.507 & 0.000 & 4.432 & 0.000 & 0.735 & 3.324 & 1.817 & 2.614 & 0.000 \\
10 & 1.507 & 0.453 & 1.507 & 0.000 & 4.432 & 0.000 & 0.735 & 3.324 & 1.817 & 2.614 & 0.000 \\
\hline
\end{tabular}

Parameter assumptions: $\beta=4.0, \alpha=0.25, \varrho=0.5, L_{2}=L_{4}=1.2$

Table 5. Tax on younger generation constant

\begin{tabular}{rlllllllllllr}
\hline$t$ & $s_{t}$ & $s_{t} / w_{t}$ & $K_{t}$ & \multicolumn{1}{c}{$I_{t}$} & $Q_{t}$ & \multicolumn{1}{c}{$I_{t} / Q_{t}$} & $r_{t}$ & $w_{t}$ & $C_{1}^{t}$ & $C_{2}^{t}$ & Comp. \\
\hline 0 & 0.942 & 0.319 & 0.942 & 0.000 & 3.940 & 0.000 & 1.046 & 2.955 & 1.314 & 2.627 & 0.000 \\
1 & 0.914 & 0.309 & 0.942 & -0.028 & 3.940 & -0.007 & 1.226 & 2.955 & 1.341 & 2.875 & -0.264 \\
2 & 0.867 & 0.309 & 0.914 & 0.126 & 4.484 & 0.028 & 0.971 & 2.803 & 1.236 & 2.292 & 0.247 \\
3 & 0.969 & 0.320 & 1.041 & -0.071 & 4.040 & -0.018 & 1.024 & 3.030 & 1.361 & 2.662 & -0.064 \\
4 & 0.950 & 0.319 & 0.969 & -0.020 & 3.969 & -0.005 & 1.039 & 2.977 & 1.327 & 2.637 & -0.018 \\
5 & 0.944 & 0.319 & 0.950 & -0.006 & 3.949 & -0.001 & 1.044 & 2.962 & 1.317 & 2.630 & -0.005 \\
6 & 0.942 & 0.319 & 0.944 & -0.002 & 3.943 & 0.000 & 1.045 & 2.957 & 1.315 & 2.628 & -0.002 \\
7 & 0.942 & 0.319 & 0.942 & 0.000 & 3.941 & 0.000 & 1.046 & 2.956 & 1.314 & 2.627 & 0.000 \\
8 & 0.942 & 0.319 & 0.942 & 0.000 & 3.941 & 0.000 & 1.046 & 2.956 & 1.314 & 2.627 & 0.000 \\
9 & 0.942 & 0.319 & 0.942 & 0.000 & 3.941 & 0.000 & 1.046 & 2.955 & 1.314 & 2.627 & 0.000 \\
10 & 0.942 & 0.319 & 0.942 & 0.000 & 3.940 & 0.000 & 1.046 & 2.955 & 1.314 & 2.627 & 0.000 \\
\hline
\end{tabular}

Parameter assumptions: $\beta=4.000, \alpha=0.25, \varrho=0.50, L_{2}=1.20, L_{4}=1.00$ 
Table 6. Transfer to older generation constant

\begin{tabular}{rlllllllllllr}
\hline$t$ & $s_{t}$ & $s_{t} / w_{t}$ & $K_{t}$ & \multicolumn{1}{c}{$I_{t}$} & $Q_{t}$ & \multicolumn{1}{c}{$I_{t} / Q_{t}$} & $r_{t}$ & $w_{t}$ & $C_{1}^{t}$ & $C_{2}^{t}$ & Comp. \\
\hline 0 & 0.942 & 0.319 & 0.942 & 0.000 & 3.940 & 0.000 & 1.046 & 2.955 & 1.314 & 2.627 & 0.000 \\
1 & 0.938 & 0.317 & 0.942 & -0.004 & 3.940 & -0.001 & 1.203 & 2.955 & 1.318 & 2.766 & -0.192 \\
2 & 0.893 & 0.317 & 0.938 & 0.134 & 4.513 & 0.030 & 0.949 & 2.821 & 1.344 & 2.441 & 0.063 \\
3 & 0.926 & 0.303 & 1.072 & -0.146 & 4.070 & -0.036 & 1.060 & 3.052 & 1.286 & 2.607 & 0.037 \\
4 & 0.937 & 0.318 & 0.926 & 0.011 & 3.924 & 0.003 & 1.050 & 2.943 & 1.306 & 2.621 & 0.011 \\
5 & 0.940 & 0.319 & 0.937 & 0.003 & 3.936 & 0.001 & 1.047 & 2.952 & 1.311 & 2.625 & 0.003 \\
6 & 0.941 & 0.319 & 0.940 & 0.001 & 3.939 & 0.000 & 1.046 & 2.954 & 1.313 & 2.626 & 0.001 \\
7 & 0.942 & 0.319 & 0.941 & 0.000 & 3.940 & 0.000 & 1.046 & 2.955 & 1.313 & 2.627 & 0.000 \\
8 & 0.942 & 0.319 & 0.942 & 0.000 & 3.940 & 0.000 & 1.046 & 2.955 & 1.314 & 2.627 & 0.000 \\
9 & 0.942 & 0.319 & 0.942 & 0.000 & 3.940 & 0.000 & 1.046 & 2.955 & 1.314 & 2.627 & 0.000 \\
10 & 0.942 & 0.319 & 0.942 & 0.000 & 3.940 & 0.000 & 1.046 & 2.955 & 1.314 & 2.627 & 0.000 \\
\hline
\end{tabular}

Parameter assumptions: $\beta=4.000, \alpha=0.25, \varrho=0.50, L_{2}=1.20, L_{4}=1.00$

Table 7. Discretionary policy

\begin{tabular}{rlllllllllllr}
\hline$t$ & $s_{t}$ & $s_{i} / w_{t}$ & $K_{t}$ & \multicolumn{1}{c}{$I_{t}$} & $Q_{t}$ & $I_{t} / Q_{t}$ & $r_{t}$ & $w_{t}$ & $C_{1}^{t}$ & $C_{2}^{t}$ & Comp. \\
\hline 0 & 0.942 & 0.319 & 0.942 & 0.000 & 3.940 & 0.000 & 1.046 & 2.955 & 1.314 & 2.627 & 0.000 \\
1 & 0.969 & 0.328 & 0.942 & 0.027 & 3.940 & 0.007 & 1.174 & 2.955 & 1.287 & 2.631 & -0.100 \\
2 & 0.978 & 0.344 & 0.969 & 0.205 & 4.550 & 0.045 & 0.887 & 2.844 & 1.428 & 2.429 & -0.014 \\
3 & 1.004 & 0.321 & 1.174 & -0.170 & 4.163 & -0.041 & 0.997 & 3.123 & 1.419 & 2.704 & -0.145 \\
4 & 0.959 & 0.319 & 1.004 & -0.044 & 4.004 & -0.011 & 1.032 & 3.003 & 1.343 & 2.649 & -0.041 \\
5 & 0.947 & 0.319 & 0.959 & -0.012 & 3.959 & -0.003 & 1.042 & 2.969 & 1.322 & 2.633 & -0.012 \\
6 & 0.943 & 0.319 & 0.947 & -0.004 & 3.946 & -0.001 & 1.045 & 2.959 & 1.316 & 2.629 & -0.003 \\
7 & 0.942 & 0.319 & 0.943 & -0.001 & 3.942 & 0.000 & 1.046 & 2.956 & 1.314 & 2.627 & -0.001 \\
8 & 0.942 & 0.319 & 0.942 & 0.000 & 3.941 & 0.000 & 1.046 & 2.956 & 1.314 & 2.627 & 0.000 \\
9 & 0.942 & 0.319 & 0.942 & 0.000 & 3.941 & 0.000 & 1.046 & 2.955 & 1.314 & 2.627 & 0.000 \\
10 & 0.942 & 0.319 & 0.942 & 0.000 & 3.941 & 0.000 & 1.046 & 2.955 & 1.314 & 2.627 & 0.000 \\
11 & 0.942 & 0.319 & 0.942 & 0.000 & 3.940 & 0.000 & 1.046 & 2.955 & 1.314 & 2.627 & 0.000 \\
\hline
\end{tabular}

Parameter assumptions: $\beta=4.000, \alpha=0.25, \varrho=0.50, L_{2}=1.20, L_{4}=1.00$ 